\title{
Evolución de la Morfología del Raquis e Inclinación Pélvica en Ciclistas de Diferentes Edades. Un Estudio Transversal
}

\author{
Evolution of Spinal Morphology and Pelvic Tilt in \\ Cyclists of Different Ages. A Cross Sectional Study \\ "José María Muyor; **Fernando Alacid; "***Pedro A. López-Miñarro \& *Antonio J. Casimiro
}

MUYOR, J. M.; ALACID, F.; LÓPEZ-MIÑARRO, P. A.; CASIMIRO, A. J. Evolución de la morfología del raquis e inclinación pélvica en ciclistas de diferentes edades. Un estudio transversal. Int. J. Morphol., 30(1):199-204, 2012.

RESUMEN: El objetivo principal del estudio fue determinar la morfología del raquis e inclinación pélvica en bipedestación en tres grupos de ciclistas de distintas edades, con un volumen de entrenamiento y experiencia similares. Un total de 65 ciclistas fueron divididos en tres grupos de edad: grupo 1 ( $\leq 30$ años; $n=20$ ); grupo 2 (entre 31 y 40 años; $n=25$ ); y grupo 3 ( $\geq 41$ años; $n=20$ ). Posteriormente se les evaluó la morfología sagital del raquis torácico, lumbar e inclinación pélvica en bipedestación, mediante el sistema Spinal Mouse. Los valores angulares medios para el raquis torácico, lumbar e inclinación pélvica para el grupo 1 fueron: 46,60 $\pm 10,09^{\circ}$; - 26,40 $\pm 7,17^{\circ} ; 13,20 \pm 4,94^{\circ}$; para el grupo $2: 47,64 \pm 8,58^{\circ} ;-26,92 \pm 6,34^{\circ} ; 12,24 \pm 4,45^{\circ}$; y para el grupo $3: 50,75 \pm 12,77^{\circ} ;-20,70 \pm$ $10,10^{\circ}$ y $8,15 \pm 8,21^{\circ}$. Se encontraron diferencias significativas en el raquis lumbar e inclinación pélvica en los grupos 1 y 2 , con respecto al 3. En conclusión, la edad es un factor que condiciona la morfología del raquis e inclinación pélvica en ciclistas que presentan los mismos años de práctica y volumen de entrenamiento en ciclismo.

PALABRAS ClAVE: Ciclismo; Postura; Cifosis; Lordosis; Pelvis; Columna vertebral.

\section{INTRODUCCIÓN}

La práctica deportiva conlleva, en muchos casos, la adopción de posturas consideradas como antinaturales para poder ejecutar un movimiento técnico preciso o mantener una posición corporal determinada en búsqueda de la mejora del rendimiento deportivo. La alteración del equilibrio fisiológico de la morfología del raquis se ha asociado con la aparición de deformidades que modifican su postura habitual (Serna et al., 1996). En este sentido, varios estudios han evaluado la influencia de la práctica de diferentes deportes sobre la morfología del raquis, encontrando una relación entre la postura predominante en los entrenamientos y/o competiciones y determinadas adaptaciones raquídeas (Alricsson \& Werner, 2006; LópezMiñarro \& Alacid, 2010; López-Miñarro et al., 2009; López-Miñarro et al. 2010; Stutchfield \& Coleman, 2006; Rajabi et al., 2008). En aquellos deportes con un elevado volumen de entrenamiento y/o predominio de posturas mantenidas o repetitivas en flexión del tronco, existe una tendencia al aumento de la cifosis torácica en bipedestación (Alricsson \& Werner; Stutchfield \& Coleman; Rajabi et al.; Wojtys et al., 2000) así como cambios morfológicos de los discos intervertebrales de la columna lumbo-sacra (Ogurkowska, 2007).

Concretamente, el ciclismo es uno de los deportes denominados como antinaturales debido a la postura mantenida del ciclista sobre la bicicleta (de Vey Mestdagh, 1998), ya que el tronco se encuentra en flexión mantenida para el apoyo de las manos sobre el manillar de la bicicleta, de modo que el raquis lumbar modifica su morfología de una lordosis en bipedestación a una inversión sobre la bicicleta (Usabiaga et al., 1997), siendo mayor la flexión intervertebral lumbar a medida que el agarre del manillar es más bajo y distal con respecto a la altura del sillín de la bicicleta (Muyor et al., 2011a, b, c).

Las posturas que mantienen el raquis en flexión, se han asociado con el aumento de la presión intradiscal del raquis torácico (Polga et al., 2004) y lumbar (Wilke et al., 1999), una mayor deformación de los tejidos espinales (Solomonow et al., 2003) y un elevado estrés vertebral (Beach et al., 2005).

* Facultad de Ciencias de la Educación. Universidad de Almería. Almería, España.

** Facultad de Ciencias del Deporte. Universidad de Murcia. Murcia, España.

**** Facultad de Educación. Universidad de Murcia. Murcia, España. 
Por otro lado, además de la práctica deportiva, la edad es un factor que influye en la morfología raquídea. En personas de mayor edad se ha encontrado un aumento de la cifosis torácica (Fon et al., 1980; Goh et al., 2000; Schwab et al., 2006), una disminución de la lordosis lumbar (Kobayashi et al., 2004; Schenkmann et al., 1996) y un menor rango de movimiento lumbar (Intolo et al., 2009; Kuo et al., 2009), en comparación con sujetos más jóvenes. Por ello, algunos estudios han evaluado la morfología del raquis en ciclistas de diferentes categorías, años de experiencia en la práctica del ciclismo y volúmenes de entrenamiento. En bipedestación, Rajabi et al. encontraron una cifosis torácica significativamente mayor en ciclistas que en sujetos no deportistas, aunque estos autores no detallan los años de experiencia y volumen de entrenamiento sobre la bicicleta. Recientemente, Muyor et al. (2011b), en ciclistas de élite con una experiencia media de 7 años de entrenamiento en ciclismo, observaron una cifosis torácica significativamente más aumentada en comparación con sujetos sedentarios. Por otro lado, Muyor et al. (2011a) al comparar ciclistas de élite con ciclistas de categoría máster 30 , con una experiencia similar en años de práctica de ciclismo, no observaron diferencias significativas en la morfología del raquis, si bien, aquellos que entrenaban más días a la semana (ciclistas de la categoría élite), presentaban un mayor porcentaje de casos con hipercifosis torácica. En un estudio posterior, Muyor et al. (2011c) encontraron un porcentaje elevado de ciclistas de categoría máster 40 con una hipercifosis torácica en bipedestación.

Debido a que en la literatura existen diversos estudios que analizan, de manera aislada, la morfología del raquis en ciclistas con una heterogeneidad de años de experiencia en ciclismo, volumen de entrenamiento y edades de los deportistas, y que la postura predominante del ciclista es en sedentación mantenida sobre la bicicleta con el raquis lumbar invertido, el objetivo del presente estudio fue determinar la morfología del raquis e inclinación pélvica en bipedestación en tres grupos de ciclistas de distintas edades, con un volumen de entrenamiento y experiencia similares.

\section{MATERIAL Y MÉTODO}

Un total de 65 ciclistas con edades comprendidas entre los 25 y 57 años, fueron divididos en tres grupos en función de su edad: grupo $1(\leq 30$ años; $\mathrm{n}=20)$; grupo 2 (entre 31 y 40 años; $\mathrm{n}=25$ ); y grupo 3 ( $\geq 41$ años; $\mathrm{n}=20$ ). Todos ellos participaron voluntariamente en el estudio. Los datos descriptivos de la muestra se presentan en la Tabla I. Los criterios de inclusión para participar en el estudio fueron: estar federados por la Real Federación Española de Ciclismo, tener un volumen de entrenamiento de 2 a 3 horas al día, con una frecuencia de 3 días a la semana, y un historial de práctica de entrenamiento ininterrumpido en ciclismo entre 5 y 8 años. Los criterios de exclusión fueron: 1) haber padecido dolor raquídeos en los 3 meses previos a la participación del estudio; 2) haber sido operado de la columna vertebral; y/o 3) tener diagnosticada alguna patología raquídea. Todos los sujetos fueron instruidos a no participar en actividades físicas o entrenamientos intensos 24 horas antes de las mediciones.

Procedimiento. El estudio fue aprobado por la Comisión de Bioética de la Universidad de Almería. Previamente a las mediciones, todos los sujetos fueron informados sobre el procedimiento y firmaron, voluntariamente, un consentimiento informado.

Para la valoración de la disposición angular de la curvas raquídeas e inclinación pélvica en bipedestación se utilizó el sistema Spinal Mouse (Idiag, Switzerland). Cada sujeto fue valorado en ropa interior, descalzo y por el mismo examinador en una misma sesión. La temperatura fue estandarizada a $24^{\circ} \mathrm{C}$. Las mediciones se realizaron en un orden aleatorio y hubo 5 minutos de descanso entre cada una de ellas.

Previamente a las mediciones, el investigador principal identificó mediante palpación y marcó, con un lápiz dérmico, el proceso espinoso de la séptima vértebra cervical (C7), así como la tercera vértebra sacra (S3). Para medir

Tabla I. Datos descriptivos de la muestra ( \pm desviación típica).

\begin{tabular}{|c|c|c|c|}
\hline & $\begin{array}{c}\text { Grupo } 1(\leq 30 \text { años }) \\
n=20\end{array}$ & $\begin{array}{c}\text { Grupo } 2 \text { (entre } 31 \text { y } 40 \text { años) } \\
n=25\end{array}$ & $\begin{array}{c}\text { Grupo } 3 \text { ( } \geq 41 \text { años) } \\
n=20\end{array}$ \\
\hline Edad (años) & $25,10 \pm 2,84$ & $35,20 \pm 2,94$ & $47,00 \pm 5,04$ \\
\hline Talla (m) & $1,78 \pm 0,07$ & $1,75 \pm 0,06$ & $1,75 \pm 0,49$ \\
\hline Masa (kg) & $73,94 \pm 8,49$ & $76,62 \pm 8,74$ & $77,03 \pm 10,16$ \\
\hline $\operatorname{IMC}(\mathrm{kg} \cdot \mathrm{m}-2)$ & $23,39 \pm 2,62$ & $25,03 \pm 2,26$ & $25,17 \pm 2,64$ \\
\hline Entrenamiento (años) & $4,85 \pm 1,75$ & $4,48 \pm 1,68$ & $5,10 \pm 1,29$ \\
\hline
\end{tabular}


las curvas raquídeas, una vez que el sujeto se colocaba en la posición a medir, se guiaba el Spinal Mouse® a lo largo de los procesos espinosos del raquis, desde $\mathrm{C} 7$ hasta $\mathrm{S} 3$. El sistema digitalizaba el contorno de la piel sobre el raquis en el plano sagital, aportando información sobre la angulación global de las curvas raquídeas. Diferentes trabajos han mostrado que el Spinal Mouse $®$ es un sistema válido y fiable para la valoración de las curvaturas raquídeas e inclinación pélvica al compararlo con técnicas radiográficas (Guermazi et al., 2006; Mannion et al., 2004; Post \& Leferink, 2004). Respecto a la curva lumbar, los valores negativos indicaron angulaciones de concavidad posterior (lordosis), mientras que los valores positivos correspondieron a una curvatura de convexidad posterior (inversión lumbar). En cuanto a la

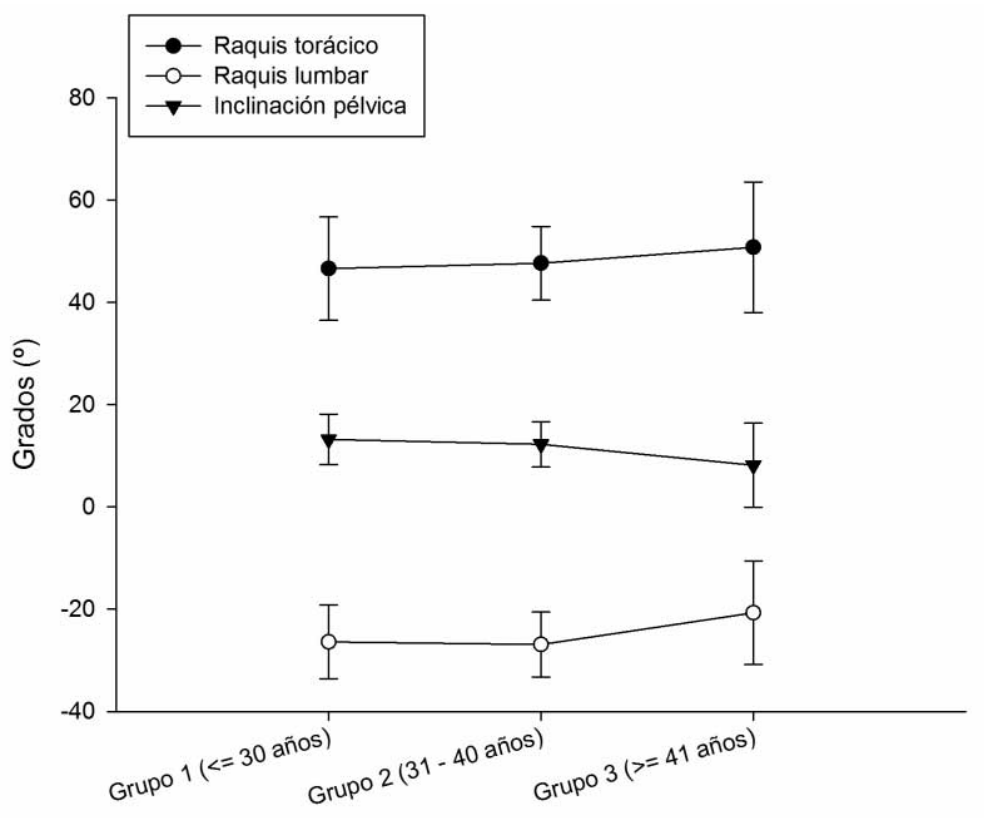

Fig. 1. Valores angulares medios ( \pm desviación típica) de la morfología del raquis e inclinación pélvica en bipedestación.

Tabla II. Comparación por pares (valor de diferencia entre las medias) para el raquis torácico, lumbar e inclinación pélvica, en las posturas evaluadas, entre los diferentes grupos de ciclistas.

\begin{tabular}{lllcc}
\hline & & & Grupo 2 & Grupo 3 \\
\hline \multirow{4}{*}{ Bipedestación } & Raquis torácico & Grupo 1 & NS $\left(1,04^{\circ}\right)$ & NS $\left(4,15^{\circ}\right)$ \\
& & Grupo 2 & - & NS $\left(3,11^{\circ}\right)$ \\
\cline { 3 - 5 } & Raquis lumbar & Grupo 1 & NS $\left(0,52^{\circ}\right)$ & $*\left(7,00^{\circ}\right)$ \\
& Inclinación & Grupo 2 & - & $*\left(6,22^{\circ}\right)$ \\
\cline { 3 - 5 } & pélvica & Grupo 1 & NS $\left(0,96^{\circ}\right)$ & $*\left(5,05^{\circ}\right)$ \\
& Grupo 2 & - & NS $\left(4,09^{\circ}\right)$ \\
\hline
\end{tabular}

$* \mathrm{p}<0,05$; NS: no significativo. Grupo $1(\leq 30$ años; $\mathrm{n}=20$ ); Grupo 2 (entre 31 y 40 años; $\mathrm{n}=25$ ); y Grupo 3 ( $\geq 41$ años; $\mathrm{n}=20$ ) posición pélvica, un valor de $0^{\circ}$ representó una posición vertical de la pelvis. Valores positivos representaron una posición de anteversión pélvica, mientras que valores negativos indicaban una posición de retroversión pélvica.

Postura. Bipedestación. En esta postura se siguió el procedimiento descrito por Muyor et al. (2011c). Los sujetos se situaban de pie, con los hombros relajados, mirada al frente, los brazos a lo largo del tronco y con una apertura de los pies igual a la anchura de sus caderas.

Análisis estadístico. La hipótesis de normalidad y homogeneidad de la varianza fue analizada mediante los test Kolmogorov-Smirnov y Levene, respectivamente. Los valores medios y desviaciones típicas fueron calculados para todas las variables. Para conocer si existían diferencias en los valores angulares del raquis torácico, lumbar e inclinación pélvica entre los tres grupos de edad de los ciclistas, se realizó un análisis de varianza (ANOVA) de un factor. Si se encontraban diferencias significativas para el efecto principal del ANOVA $(\mathrm{p}<0,05)$, se realizó una comparación por pares mediante un análisis post hoc con ajuste de Bonferroni. Los datos fueron analizados usando el software SPSS, v. 15.0 y el nivel de significación a priori fue de $\mathrm{p}$ $<0,05$.

\section{RESULTADOS}

Los valores medios y desviaciones típicas del raquis torácico, lumbar e inclinación pélvica en bipedestación, en los tres grupos de edad, se presentan en la Figura 1. El raquis torácico mostró una tendencia hacia un incremento de la flexión intervertebral a medida que aumentaba la edad de los ciclistas, si bien no se encontraron diferencias significativas (Tabla II). En cuanto al raquis lumbar, el grupo de ciclistas de mayor edad $(\geq 41$ años) presentó una significativa menor la lordosis e inclinación pélvica con respecto a los otros dos grupos de ciclistas (Tabla II).

\section{DISCUSIÓN}

El objetivo principal del estudio fue determinar la morfología del raquis e inclinación pélvica en bipedestación en tres grupos de ciclistas de diferentes edades, con los mismos años de 
práctica en ciclismo y un similar volumen de entrenamiento semanal y diario sobre la bicicleta.

El principal hallazgo del estudio fue que a medida que aumenta la edad de los ciclistas, hay un incremento de la cifosis torácica en bipedestación, aunque sin diferencias significativas entre los grupos. En estudios previos, Rajabi et al. observaron, en ciclistas, un morfotipo torácico significativamente más aumentado en comparación con sujetos sedentarios. Estos autores argumentaron que sus resultados eran debidos a la adaptación raquídea, por la postura en flexión del tronco mantenida por el ciclista para alcanzar el agarre del manillar. En estudios recientes, Muyor et al. (2011a, 2011b, 2011c) han observado, en ciclistas de diferentes categorías y en sujetos no deportistas (Muyor et al., 2011b), que el raquis torácico se mantiene más rectificado sobre la bicicleta con las manos apoyadas en los tres agarres más utilizados en ciclismo (agarre transversal, de manetas y bajo) que en la postura de bipedestación relajada, debido a la aducción escapular y retropulsión de hombros generada al apoyar las manos y descargar el peso del tronco sobre el manillar. Por ello, estos autores concluyen que los elevados porcentajes de ciclistas con hipercifosis torácica encontrados en bipedestación, podrían deberse más a otros factores que a la práctica del ciclismo.

En el presente estudio, los ciclistas con más de 40 años mostraron una significativa menor lordosis lumbar y anteversión pélvica con respecto a los ciclistas más jóvenes. En un trabajo reciente, realizado en ciclistas de la categoría máster 40, con 12 años de experiencia en ciclismo, Muyor $e t$ al. (2011c) encontraron que más del $30 \%$ de los ciclistas analizados presentaban una rectificación lumbar. Estos autores asociaron esta disminución de la lordosis lumbar a una adaptación vertebral a la flexión continua del raquis lumbar sobre la bicicleta, ya que éste se dispone en inversión (Usabiaga $e t$ al., 1997). El volumen de entrenamiento desarrollado en diferentes disciplinas deportivas, parece ser una variable importante en las adaptaciones de la morfología raquídea. En este sentido, Wojtys et al. encontraron un incremento propor- cional de la cifosis torácica en relación al tiempo de entrenamiento por año (horas/años). Sin embargo, la lordosis lumbar en bipedestación no mostró modificaciones hasta que no se superaban las $400 \mathrm{~h} / \mathrm{año}$ de entrenamiento. Alricsson \& Werner observaron un incremento de la cifosis torácica tras 5 años de entrenamiento intenso en esquí de fondo. Öztürk et al. (2008) encontraron en 70 ex-jugadores de fútbol con una experiencia deportiva de 11 años, una mayor degeneración vertebral y una significativa disminución de la altura de los discos intervertebrales que respecto a personas de la misma edad que no practicaban deporte. En el presente estudio, los tres grupos de ciclistas presentaban los mismos años de práctica en ciclismo y volumen de entrenamiento diario y semanal sobre la bicicleta, por lo que la influencia del ciclismo sobre el raquis era la misma para todos los sujetos estudiados.

La edad es considerada como un factor influyente en la configuración de la morfología raquídea. Sujetos con edades más avanzadas han mostrado un aumento de la cifosis torácica (Fon et al.; Goh et al.; Schwab et al.), disminución de la lordosis lumbar (Kobayashi et al., 2004; Schenkmann et al., 1996) y una mayor horizontalización del sacro (RuizIbán et al., 2005). Todas estas circunstancias se han observado en los ciclistas analizados en el presente estudio, puesto que aquellos de mayor edad (> 40 años) presentaron valores ligeramente superiores de cifosis torácica y una significativa menor lordosis lumbar y anteversión pélvica en bipedestación con respecto a los ciclistas más jóvenes. Debido a la estrecha relación del raquis lumbo-pélvico, en otros trabajos se han observado que generalmente los sujetos que presentan una menor inclinación pélvica anterior, desarrollan una rectificación de la lordosis lumbar en bipedestación (Day et al., 1984; Levine \& Whittle, 1996).

En conclusión, en ciclistas con los mismos años de experiencia y volumen de entrenamiento diario y semanal sobre la bicicleta, se observa que a medida que avanza la edad, existe una tendencia al incremento de la cifosis torácica, pérdida de la lordosis lumbar y menor inclinación pélvica anterior.

MUYOR, J. M.; ALACID, F.; LÓPEZ-MIÑARRO, P. A.; CASIMIRO, A. J. Evolution of spinal morphology and pelvic tilt in cyclists of different ages. A cross sectional study. Int. J. Morphol., 30(1):199-204, 2012.

SUMMARY: The aim of this study was to evaluate the sagittal spinal morphology and pelvic tilt in standing in three different age-group cyclists who had the same cycling experience and training volume. A total of 65 cyclists were divided in three age-groups: group 1: ( $\leq 30$ years old; $n=20$ ); group 2 (between 31 y 40 years old; $n=25)$; and group $3(\geq 41$ years old; $n=20)$. The Spinal Mouse was used to measure sagittal thoracic and lumbar spine and pelvic tilt in standing on the floor. The mean values for thoracic and lumbar spine and pelvic tilt were: group $1,46.60 \pm 10.09^{\circ}$; $-26.40 \pm 7.17^{\circ} ; 13.20 \pm 4.94^{\circ}$; group $2,47.64 \pm 8.58^{\circ} ;-26.92 \pm 6.34^{\circ} ; 12.24 \pm 4.45^{\circ}$; and group $3,50.75 \pm 12.77^{\circ} ;-20.70 \pm 10.10^{\circ}$ y $8.15 \pm 8.21^{\circ}$, respectively. Significant differences were found for the lumbar spine and pelvic tilt in the groups 1 and 2 with respect to group 3 . In conclusion, aging is a conditional factor to the sagittal spinal morphology and pelvic tilt in cyclists who present the same years of experience and training volume in cycling.

KEY WORDS: Cycling; Posture; Kyphosis; Lordosis; Pelvis; Spine. 


\section{REFERENCIAS BIBLIOGRÁFICAS}

Alricsson, M. \& Werner, S. Young elite cross-country skiers and low back pain. A 5-year study. Phys. Ther. Sport, 7:181-4, 2006.

Beach, T. A.; Parkinson, R. J.; Stothart, J. P. \& Callaghan, J. P. Effects of prolonged sitting on the passive flexion stiffness of the in vivo lumbar spine. Spine J., 5:145-54, 2005.

Day, J. W.; Smidt, G. L. \& Lehmann, T. Effect of pelvic tilt on standing posture. Phys. Ther., 64:510-6, 1984.

de Vey Mestdagh, K. Personal perspective: in search of an optimum cycling posture. Appl. Ergon., 29:325-34, 1998.

Fon, G.; Pitt, M. J. \& Thies, A. C. Jr. Thoracic kyphosis: a range in normal subjects. Am. J. Roentgenol., 134:979-83, 1980.

Goh, S.; Price, R. I.; Leedman, P. J. \& Singer, K. P. A comparison of three methods for measuring thoracic kyphosis: implications for clinical studies. Rheumatology, 39:310-5, 2000.

Guermazi, M.; Ghroubi, S.; Kassis, M.; Jaziri, O.; Keskes, H.; Kessomtini, W.; Hammouda, I. B. \& Elleuch, M. H. Validity and reliability of Spinal Mouse ${ }^{\circledR}$ to assess lumbar flexion. Ann. Readapt. Med. Phys., 49:172-7, 2006.

Intolo, P.; Milosavljevic, S.; Baxter, D. G.; Carman, A. B.; Pal, P. \& Munn, J. The effect of age on lumbar range of motion: A systematic review. Man. Ther., 14:596-604, 2009.

Kobayashi, T.; Atsuta, Y.; Matsuno, T. \& Takeda, N. A longitudinal study of congruent sagittal spinal alignment in an adult cohort. Spine, 29:671-6, 2004.

Kuo, Y.; Tully, E. A. \& Galea, M. P. Video based measurement of sagittal range of spinal motion in young and older adults. Man. Ther., 14:618-22, 2009.

Levine, D. \& Whittle, M. W. The effects of pelvic movement on lumbar lordosis in the standing position. J. Orthop. Sports Phys. Ther., 24:130-5, 1996.

López-Miñarro, P. A. \& Alacid, F. Influence of hamstring muscle extensibility on spinal curvatures in young athletes. Sci. Sports, 25:88-93, 2010.

López-Miñarro, P. A.; Alacid, F. \& Muyor, J. M. Comparación del morfotipo raquídeo y extensibilidad isquiosural entre piragüistas y corredores. Rev. Int. Med. Cienc. Act. Fís. Deporte, 9:379-92, 2009.

López-Miñarro, P. A.; Muyor, J. M. \& Alacid, F. Sagittal spinal curvatures and pelvic tilt in elite young kayakers. Med. Sport, 63:509-19, 2010.

Mannion, A. F.; Knecht, K.; Balaban, G.; Dvorak, J. \& Grob, D. A new skin-surface device for measuring the curvature and global and segmental ranges of motion of the spine: reliability of measurements and comparison with data reviewed from the literature. Eur. Spine J., 13:122-36, 2004.

Muyor, J. M.; López-Miñarro, P. A. \& Alacid, F. Spinal posture of thoracic and lumbar spine and pelvic tilt in highly trained cyclist. J. Sports Sci. Med., 10:355-61, 2011a.

Muyor, J. M., López-Miñarro, P. A. \& Alacid, F. A comparison of the thoracic spine in the sagittal plane between elite cyclists and non-athlete subjects. J. Back Musculoskelet. Rehabil., 24:129-35, 2011b.

Muyor, J. M.; Alacid, F. \& López-Miñarro, P. A. Spinal Posture of Thoracic and Lumbar Spine in Master 40 Cyclists. Int. J. Morphol., 29:727-32, 2011c.

Ogurkowska, M. B. Pathological change of intervertebral disc of the lumbosacral spine of competitive rowers. Biol. Sport, 24:375-88, 2007.

Öztürk, A.; Özkan, Y.; Özdemir, R.; Yaçin, N.; Akgöz, S.; Saraç, V. \& Aykut, S. Radiographic changes in the lumbar spine in former professional football player: a comparative and matched controlled study. Eur. Spine J., 17:136-41, 2008.

Polga, D. J.; Beaubien, B. P.; Kallemeier, P. M.; Schelhas, K. P.; Lee, W. D.; Buttermann, G. R. \& Wood, K. B. Measurement of in vivo intradiscal pressure in healthy thoracic intervertebral disc. Spine, 29:1320-4, 2004.

Post, R. B. \& Leferink, V. J. Spinal mobility: sagittal range of motion measured with the SpinalMouse, a new non-invasive device. Arch. Orthop. Trauma Surg., 124:187-92, 2004.

Rajabi, R.; Doherty, P.; Goodarzi, M. \& Hemayattalab, R. Comparison of thoracic kyphosis in two groups of elite GrecoRoman and free style wrestlers and a group of non-athletic subjects. Br. J. Sports Med., 42:229-32, 2008.

Ruiz-Ibán, M.; Elías-Martín, M. E. \& Ruiz-Fernández, J. A. Inclinación pélvica en el plano sagital. Descripción y validación de un nuevo sistema de medida. Valores normales en 75 sujetos. Rehabil., 39:121-7, 2005.

Schenkmann, M.; Shipp, K. M.; Chandler, J.; Studenski, S. A. \& Kuchibhatla, M. Relationships between mobility of axial structures and physical performance. Phys. Ther., 76:276-85, 1996.

Schwab, F.; Lafage, V.; Boyce, R.; Skalli, W. \& Farcy, J. P. Gravity line analysis in adult volunteers. Age-related correlation with spinal parameters, pelvic parameters and foot position. Spine, 31:E959-67, 2006.

Serna, L.; Santonja, F. \& Pastor, A. Exploración clínica del plano sagital del raquis. Selección, 5:88-102, 1996. 
MUYOR, J. M.; ALACID, F.; LÓPEZ-MIÑARRO, P. A.; CASIMIRO, A. J. Evolución de la morfología del raquis e inclinación pélvica en ciclistas de diferentes edades. Un estudio transversal. Int. J. Morphol., 30(1):199-204, 2012.

Solomonow, M.; Zhou, B. H.; Baratta, R. V. \& Burger, E. Biomechanics and electromyography of accumulative lumbar disorder: response to static flexion. Clin. Biomech., 18:890-8, 2003.

Stutchfield, B. M. \& Coleman, S. The relationships between hamstring flexibility, lumbar flexion, and low back pain in rowers. Eur. J. Sports Sci., 6:255-60, 2006.

Usabiaga, J.; Crespo, R.; Iza, I.; Aramendi, J.; Terrados, N. \& Poza, J. J. Adaptation of the lumbar spine to different positions in bicycle racing. Spine, 22:1965-9, 1997.

Wilke, H. J.; Neef, P.; Caimi, M.; Hoogland, T. \& Claes, L. E. New in vivo measurements of pressures in the intervertebral disc in daily life. Spine, 24:755-62, 1999.

Wojtys, E. M.; Ashton-Miller, J. A.; Huston, L. J. \& Moga, P. J. The association between athletic training time and sagittal curvature of the immature spine. Am. J. Sports Med., 28:4908,2000 .

\section{Dirección para correspondencia: \\ Dr. José María Muyor \\ Universidad de Almería \\ Área Didáctica de la Expresión Corporal \\ Edificio Central, Despacho 0.47 \\ Ctra. Sacramento S/N, 04120. \\ La Cañada de San Urbano, Almería \\ ESPAÑA}

Teléfono: 34950014045

Email: josemuyor@ual.es

Recibido : 14-07-2011

Aceptado: 09-11-2011 